\title{
New Gamma-Ray Probe of the Baryonic Dark Matter
}

\author{
Anatoli lyudin* \\ MPE, Garching, Germany, and Moscow State Univ., Skobeltsyn Insitute of Nuclear Physics, \\ Moscow, Russia \\ E-mail: ani@mpe.mpg.de, aiyudinesrd.sinp.msu.ru

\section{Vadim Burwitz} \\ Max-Planck-Institut für extraterrestrische Physik, Postfach 1312, 85741 Garching, Germany \\ E-mail: burwitz@mpe.mpg.de

\section{Jochen Greiner} \\ Max-Planck-Institut für extraterrestrische Physik, Postfach 1312, 85741 Garching, Germany \\ E-mail: jcg@mpe.mpg.de
}

\section{Guido DiCocco}

IASF-Bologna, Bologna, Italy

E-mail: dicocco@bo.iasf.cnr.it

\section{Stefan Larsson}

Stockholm University, Stockholm, Sweden

E-mail: stefaneastro.su.se

We expand on the recently reported detections of the gamma-ray resonant absorption along the line of sight toward gamma-ray bright quasars (QSOs), like 3C279. We propose to use this novel gamma-ray absorption method to study the Dark Matter distribution in the Milky Way, as well as in the Local Group.

Properties of the absorber that was detected on the sight lines towards gamma-ray bright QSOs at zero redshift are discussed. We compare our results with the expected Dark Matter distributions in the halo of Milky Way, that were simulated in boundaries of different CDM models.

Application of this new method to study evolution of CDM in the QSO host galaxies, and of baryons distribution in the halo of galaxies in the Local Universe is proposed.

Baryons in Dark Matter Halos

5-9 October 2004

Novigrad, Croatia

\footnotetext{
*Speaker.
} 


\section{Introduction}

One of the pressing problems in the modern cosmology is that the nature of the dark matter is at present unknown. The properties of dark matter on large scales are compatible with a heavy, collision-less species which clusters gravitationally, cold dark matter (CDM). But there are inconsistencies between simulations and observations of the dark matter halo of galaxies in boundaries of CDM models. While the outer slope derived from the numerical simulations [9], [10], [12], [18], and [19] is consistent with current observations, there are problems with fitting the inner slope of halos. Observations of rotation curves of many dwarf galaxies, indicating almost constant density cores, suggest that the inner density profiles in these systems are much shallower than found in simulations [4], [5], and [22].

We propose to use a new method, namely, a recently introduced $\gamma$-ray probe of the absorbing columns on the line sights towards bright quasars (QSOs) [14] to solve the discrepancy between observations and simulations of the halo density profiles. The $\gamma$-ray absorption can be used to probe higher column densities than those accessible for the longer wavelengths. Additionally, it is not sensistive to the absorber ionization or chemical state.

In our method we rely on the photoabsorption processes that happen on the nuclei. Apart of the well known high-energy photons attenuation processes, like Compton scattering and pair production, with a rather smooth energy dependence of the cross section at $\mathrm{E}_{\gamma} \geq 100 \mathrm{keV}$ [13], there are three more photoabsorption processes to mention [14]. Namely, the photoabsorption cross-section on nuclei have three resonant-like peaks in the cross section, at energies of $\sim 7 \mathrm{MeV}$ ("pygmy" dipole resonance (PDR)), 20-30 MeV (giant dipole resonance (GDR)), and $\sim 325 \mathrm{MeV}$ ( $\Delta$-resonance) [1]. The best studied of these three processes are GDR, and $\Delta$-isobar resonance [1].

From the ratio of the typical absorption cross sections in the $\gamma$-ray and X-ray regimes follows that $\gamma$-rays probe the column densities of the order of $\sim 10^{26} \mathrm{~cm}^{-2}$ via the resonant $\gamma$-absorption, while in X-ray regime we probe column densities of the order of $\leq 10^{25} \mathrm{~cm}^{-2}$.

\section{Absorbing columns near Galactic Center}

Results used to constrain the shape of the baryonic matter distribution in the region of Galactic Center (GC) are based on the data acquired by the gamma-ray telescopes COMPTEL and EGRET on-board of the Compton Gamma-Ray Observatory (CGRO). A detailed description of the instruments and data analysis is given in [21] for COMPTEL, and in [23], and [16] for EGRET.

We analysed COMPTEL data using the maximum-likelihood method (SRCFIX), that evolved from the work on diffuse emission modeling [24]. The analysis of EGRET data is also based on the maximum likelihood analysis of the observed region [16].

In this paper we will mostly use results of EGRET, that has best sensitivity near the $300 \mathrm{MeV}$, i.e near the energy of the $\Delta$-resonance absorption for systems with a small redshift. The differential photon flux from AGN at the Earth can be written as a function of the photon energy and of redshift: $\frac{d N}{d E}=\left(\frac{d N}{d E}\right)_{\text {unabsorbed }} \cdot e^{-\tau(E, z)}$.

The dependence of $\tau$ on $E$ and $z$ is quite complex, to simplify it we assume that we are dealing with two absorbers, one in the QSO host galaxy, and the second absorber in the Milky Way. 

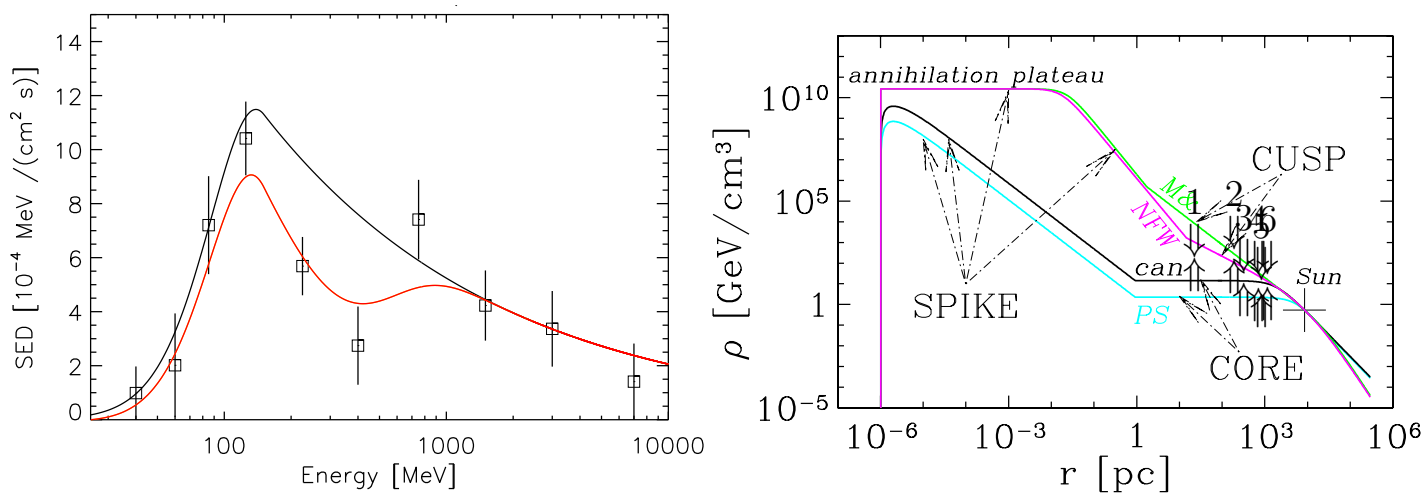

Figure 1: Left: A fi t to the 3EG J1744-3011 time-averaged SED, that includes the $\Delta$ resonance photon absorption (red line) in the matter of Milky Way. Black line shows "Band"-function fi t. Right: The comparison of the DM density profi les derived from the numerical simulations, adapted from [6], with the preliminary normalized values of the baryonic absorption columns derived from the amplitude of the $\Delta$ resonance photon absorption troughs in the SEDs of EUIDs listed in the Table 1. Here "can" stands for canonical distribution [2]; "PS" stands for distribution of [20]; "M\&" stands for [17], and "NFW" is from [18].

As a first step of a SED analysis, we fit a smooth function to the spectrum, which we choose to be the so-called "Band"-function [3], that describes well not only power-law spectra, but also spectra with a break. In the second fit we use as a fit function the sum of the "Band"-function and a gaussian. The significance of the fit improvement we evaluate using the probability for the spurious improvement of the fit from the value of $\Delta \chi^{2}=\chi_{\text {band }}^{2}-\chi_{\text {band }+ \text { gauss }}^{2}$ for 3 d.o.f. as a test for such an improvement, similar to [8]. Some of the $\gamma$-ray bright sources in the GC region, can be used to probe the baryonic matter distribution in the center of Milky Way. The very first results of such a "probe" are discussed below. We have used SEDs of 6 EGRET Unidentified sources (EUIDs)

\begin{tabular}{||c||c||c||c||c||c||c||c||}
\hline Object & $l^{o}$ & $d^{o}$ & $\theta_{95}$, deg. & $\Delta d,(\mathrm{pc})$ & $N_{H} \times 10^{26}$ & $N_{H}{ }^{*}$ & No. \\
\hline \hline 3EG J1746-2851 & 00.11 & -0.04 & 0.13 & 17.366 & $24_{-10}^{+6}$ & $6.5_{-2.7}^{+1.6}$ & 1 \\
\hline 3EG J1744-3011 & -1.15 & -0.52 & 0.32 & 187.26 & $35_{-23}^{+29}$ & $9.5_{-6.2}^{+7.8}$ & 2 \\
\hline 3EG J1736-2908 & -1.21 & 1.56 & 0.62 & 275.69 & $4.1 \pm 2.4$ & $1.11 \pm 0.65$ & 3 \\
\hline 3EG J1741-2312 & 04.42 & 3.76 & 0.57 & 813.02 & $3.8 \pm 2.5$ & $1.02 \pm 0.68$ & 5 \\
\hline 3EG J1717-2737 & -2.33 & 5.95 & 0.64 & 895.92 & $3.1 \pm 2.2$ & $0.84 \pm 0.59$ & 6 \\
\hline 3EG J1741-2050 & 06.44 & 5.00 & 0.63 & 1146.1 & $3.9 \pm 2.4$ & $1.07 \pm 0.65$ & 7 \\
\hline
\end{tabular}

${ }^{*}$ Value normalized to the mean value of $N_{H}=(3.70 \pm 1.19) \times 10^{26} \mathrm{~cm}^{-2}$ for $\Delta \mathrm{d}>275 \mathrm{pc}$.

Table 1: EUIDs, their projected distance from the GC, and absorbing columns for these projected distances.

[11] to derive baryonic absorbing columns near GC (Table 1). 3EG J1756-2851 is at a redshift of $\sim 0.85$ [14]. By analogy with 3EG J1736-2908 which was identified with the Seyfert I galaxy GRS 1734-292 [7], and [15], we consider 4 other EUIDs also as extragalactic sources. For 5 out of 6 EUIDs the fit and the derived absorbing column was made in the region of $\Delta$-resonance (Fig. 1, left). Only for 3EG J1746-2851 we have used results of the fit at the energies of GDR, e.g around $25 \mathrm{MeV}$. In this case we derived the lowest possible value of the absorbing column. Four columns 
derived for EUIDs at the projected distances of $\Delta d>275$ pc were used to calculated weighted mean value of the absorbing column, that was normalized to the calculated DM density profiles of Fig. 1 (right) at $r=1 \mathrm{kpc}$. Figure 1 (right) shows the Milky Way central core/cusp profiles derived by the numerical simulations that are (rather crudely) compared with the baryonic absorbing columns measured at different projected distances from the GC.

The incentive of our paper was to demonstrate the potential of the $\gamma$-ray absorption method for this or similar studies. Still, already these results give a hint of a more shallow slope of the inner cusp than that from the numerical simulations [17], or [18]. It is clear that more sensitive measurements of EUID SEDs in the GC region are needed to provide definite conclusion on the preferable baryonic matter profile close to the Galactic Center. Such measurements can be performed by the forthcoming new gamma-ray mission GLAST, that will be launched in 2007, see http://www-glast.slac.stanford.edu, for the GLAST description.

\section{References}

[1] Ahrens, J., Nucl.Phys A446, 229c (1985).

[2] Bahcall, J.N., and Soneira, R.M., ApJS 44, 73 (1980).

[3] Band, D., Matteson, J., Ford, L., et al., ApJ 413, 281 (1993).

[4] de Blok, W.J.G., McGaugh, S.S., Bosma, A. and Rubin, V.C., ApJ 552, L23 (2001).

[5] van den Bosch, F.C., Robertson, B.E., Dalcanton, J.J. and de Blok, W.J.G., AJ 119, 1579 (2000).

[6] Buckley, J., Burnett, T., Sinnis, G., et al., [astro-ph/0201160] (2002).

[7] Di Cocco, G., Foschini, L., Grandi, P., et al., [astro-ph/0406300] (2004).

[8] Freeman, P.E., Graziani, C., Lamb, D.Q., et al., ApJ 524, 753 (1999).

[9] Fukushige, T., Kawai, A. and Makino, J., ApJ 606, 625 (2004).

[10] Ghigna, S., Moore, B., Governato, F., Lake, G., Quinn, T. and Stadel, J., ApJ 544, 616 (2000).

[11] Hartman, R.C., Bertch, D.L., Bloom, S.D., et al., ApJS 123, 79 (1999).

[12] Hayashi, E., et al., astro-ph/0310576 (2003).

[13] Hubbell, J.H., Atomic Data 3, 241 (1971).

[14] Iyudin, A.F., et al., $A \& A$, submitted (2004).

[15] Martí, J., Mirabel, I.F., Chaty, S., Rodríguez, L.F., A\&A 330, 72 (1998).

[16] Mattox, J.R., Bertsch, D.L., Chiang, J., et al., ApJ 461, 396 (1996).

[17] Moore, B., Governato, F., Quinn, T., Stadel, J., and Lake, G., ApJ 499, L5 (1998).

[18] Navarro, J.F., Frenk, C.S. and White, S.D.M., ApJ 490, 493 (1997).

[19] Navarro, J.F., et al., MNRAS 349, 1039 (2004).

[20] Persic, M., Salucci, P., and Stel, F., MNRAS 281, 27 (1996).

[21] Schönfelder, V., Aarts, H., Bennett, K., et al., ApJS 86, 657 (1993).

[22] Simon, J.D., Bolatto, A.D., Leroy, A. and Blitz, L., astro-ph/0310193 (2003).

[23] Thompson, D.J., Bertsch, D.L., Fichtel, C.E., et al., ApJS 86, 629 (1993).

[24] van Dijk, R., PhD Thesis, Amsterdam Univ. (1996). 